MATEC Web of Conferences 40, 03001 (2016)

DOI: $10.1051 /$ matecconf/20164003001

(C) Owned by the authors, published by EDP Sciences, 2016

\title{
Kinematic Performances of a Novel TLPM Parallel Robot
}

\author{
Jianxun Fu, Feng Gao \\ School of Mechanical Engineering, Shanghai Jiao Tong University, Shanghai, China
}

\begin{abstract}
This paper investigates the kinematic performances of kinematics, Jacobian, singularity and interferences of a novel six-degree-of freedom (DOF) parallel manipulator. Analytical solutions of the forward position kinematics have been worked out. Three-dimensional Cartesian space generated by a stroke interval from $l_{\min }$ to $l_{\max }$ fulfils the point workspace are illustrated, and the reachable workspaces are obtained. The notion of pure translational Jacobian of constraint matrix is introduced, and two types of conventional singularities are analyzed. Finally, auxiliary vectors are introduced to determine the link interferences, shown that there are two kinds of interferences in the system, one is angle-interference in one limb, and the other is distance-interference in adjacent limbs.
\end{abstract}

\section{Introduction}

A parallel robot is defined as a mechanism having at least two kinematics chains connecting the base to the end effector, and the end effector has $n \leq 6$ DOF [1]. Certain parallel mechanisms have shown to offer many advantages over serial mechanisms, such as high stiffness, large payload capacity, compact structure, low inertia, high accuracy, etc[2]. Recently a large number of PMs have been built and several distinctive designs and analysis approaches have been introduced $[3,4,5]$.

A key issue for parallel robot is optimal design. Optimal design methodologies have to rely on kinematic performance indices. There is a rich body of literatures addressing the design problem of developing good performance of a manipulator based upon workspace optimization $[6,7]$.

The kinematic performances of a manipulator, Jacobian and singularity analyses are commonly used $[8,9]$. Much research has been devoted to the study of mechanical these criterias of a parallel mechanism $[10,11,12]$. Firmani and Podhorodeski [13] described singularity of planar parallel manipulators based on forward kinematic solutions. The workspace analysis has continued to attract substantial attention over the years.

The performance analysis is an important activity during the design process of robotics systems. The performance analysis determine influence of variations in the geometric parameters. A good design is one in which a manipulator of suitable mechanical possesses good performances. Performance criterias are commonly used in structural design [14]. The optimum design and selection of the geometric parameters is very important in robotics In general, high performance is given by high mechanical advantage. The relative importance of these aspects of performance depends on the individual design application. Although many design approaches have been proposed for this problem, much effort on it continues.

\section{Structural description of the Proposed Robot}

\subsection{The characteristics of the TLPM}

The TLPM robot has a parallel kinematic structure with three identical branches connecting the fixed platform to the moving platform. Each branch is configured by two passive revolute (R) joints, an active $\mathrm{R}$ joint, an active prismatic $(\mathrm{P})$ joint and a universal $(\mathrm{U})$ joint. This design has six DOF, namely three translations along $\mathrm{x}, \mathrm{y}$ and $\mathrm{z}$ directions and three rotations around $\mathrm{x}, \mathrm{y}$ and $\mathrm{z}$ axes. One of the advantages of this structure is that three translational and three rotational motions are decoupled, the end-effector position is only determined by three lengths of prismatic actuators, while the rotational angles are relative to all six inputs, so that it is easier to computer the end-effector position and orientation of the manipulator in inverse and forward kinematics. In this paper, we only consider the positon performances of the proposed robot.

\subsection{Geometric parameters of the TLPM}

According to the mechanical structure design, each limb has been assembled on the base platform at an angle $\beta_{1}$. The axes of $\mathrm{R}$ joints and the guide directions of the three $P$ joints intersect at a common point $p$.

The $i^{\text {th }}, i=1,2,3$, limb connects to the base platform through a universal joint, and $\mathbf{q}_{i}, i=1,2,3$, denotes the point where the two axes of the $U$ joint intersect at the 
base platform. The points $\mathbf{q}_{1}, \mathbf{q}_{2}$, and $\mathbf{q}_{3}$ lie evenly on a circle of radius $L$, and the $U$ joints are tangent to the circle.

In this study, the geometric design parameters of the $i^{\text {th }}$ limb are defined. Angle $\alpha_{1}$ denotes the angle between the axis of the $\mathrm{P}$ joint and the axis of R1 joint, $\alpha_{2}$ denotes the angle between the axis of the R1 joint and the axis of the R2 joint, $\beta_{2}$ is defined from the geometric centre of the line from the moving platform to the axis of the R2 joint and $L$ is the radius of the base platform. Angles $\eta_{i 1}$, $i=1,2,3$, are defined as the angles between the projection of the axes of the actuated $\mathrm{P}$ joints on the base platform and a given reference in that plane in the state of the initial configuration. Angles $\eta_{i 2}, i=1,2,3$, are defined between the projection of the axis of the R2 joint of the $i^{\text {th }}$ limb on the moving platform and a given reference in that plane, as shown in Fig. 1. The input parameters $l_{i}, i=1,2$, 3 , respectively denote the actuator angle and length with respect to the initial values.

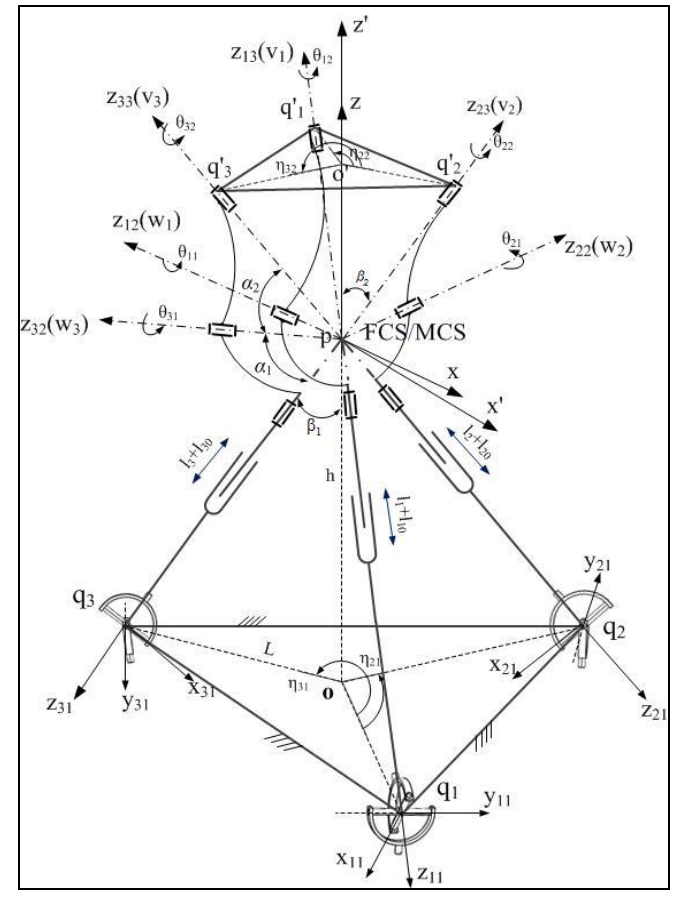

Figure 1. Kinematic diagram of the TLPM

\section{Forward position analysis}

For convenient analysis, a fixed coordinate system (FCS) is fixed to the base platform; its origin always fixed at the initial position of the point $p$. Its $z$ axis is defined as the axis in the direction from $o$ to $p$ and its $x$-axis is parallel to the vector $\mathbf{o q}_{1}$. A moving coordinate system (MCS) is located at the point $p$ and is fixed to the moving platform. Its $z^{\prime}$ axis is defined in the direction from the $o$ to $p$, and its $x^{\prime}$ axis is parallel to the $x$ axis.

As previously mentioned, each link has been set up on the base platform with a certain angle, and the fixed points $\mathbf{q}_{i}$, are uniformly distributed on the base platform. Therefore, the bases of the limbs constitute an equilateral triangle. Here we denote $c$ to abbreviation cosine and $\mathrm{s}$ for sine. Referring to Fig. 1, the position vectors of point $\mathbf{q}_{i}$ with respect to the FCS are expressed as

$$
\mathbf{q}_{i}=\mathbf{R}_{\eta_{i 1}}\left(\begin{array}{lll}
L & 0 & -h
\end{array}\right)^{T}
$$

where $\mathbf{R}_{\eta_{1 i}}=\left[\begin{array}{ccc}\mathrm{c} \eta_{i 1} & -\mathrm{s} \eta_{i 1} & 0 \\ \mathrm{~s} \eta_{i 1} & \mathrm{c} \eta_{i 1} & 0 \\ 0 & 0 & 1\end{array}\right]$.

Because the base platform is an equilateral triangle, the position vectors $\mathbf{q}_{i}=\left(x_{i}, y_{i}, z_{i}\right)^{T}$. can be calculated with Eq. (1); the Cartesian coordinates of the position moving platform are denoted as $\mathbf{p}=(x, y, z)^{T}$. There exist at most three lines in the base that satisfy three distances between the point $p$ and the $i^{\text {th }} \mathrm{U}$ joint :

$$
\left\|\mathbf{p}-\mathbf{q}_{i}\right\|=\left(l_{i 0}+l_{i}\right), i=1,2,3
$$

Where $l_{i 0}$-The initial length of the limb, and $l_{l}$-Input length of the $i^{\text {th }}$ parametric joint.

Eqs. (2) are the foundation equations for the solution of kinematics problem in this paper. For a given position of the end-effector, one solution for lengths of the prismatic joints $l_{\mathrm{i}}, i=1,2,3$, is obtained. The solution of the inverse kinematics issue is thereby complete.

The forward kinematics solution is shown at the intersection of the point $\mathrm{p}$ created by the length of the prismatic actuators $l_{i}$. According to the above Eq. (2), by substitution of the points $p$ and qi, the forward kinematic problem can be formulated as in the following equations:

$$
\begin{aligned}
& x=-l_{1}^{2} / 3 L- 2 L_{0} l_{1} / 3 L+l_{2}^{2} / 6 L+L_{0} l_{2} / 3 L+ \\
& l_{3}^{2} / 6 L+L_{0} l_{3} / 3 L \\
& y=\sqrt{3} l_{3}^{2} / 6 L+\sqrt{3} L_{0} l_{2} / 3 L-\sqrt{3} L_{0} l_{2}^{2} / 6 L \\
&-\sqrt{3} L_{0} l_{2} / 3 L \\
& z^{(1)}=-h+\sqrt{\left(L_{0}+l_{1}\right)^{2}-(X-L)^{2}-Y^{2}} \\
& z^{(2)}=-h-\sqrt{\left(L_{0}+l_{1}\right)^{2}-(X-L)^{2}-Y^{2}}
\end{aligned}
$$

Table 1. Two real solutions for the position vector of the moving platform

\begin{tabular}{|c|c|}
\hline $\begin{array}{c}\text { Cartesian } \\
\text { positions }\end{array}$ & Positional variables(mm) \\
\hline $\mathbf{P}_{1}$ & $(100,100,100)$ \\
\hline $\mathbf{P}_{2}$ & $(100,100,-1280)$ \\
\hline
\end{tabular}

Eqs. (3-6) are the polynomial solution for the forward kinematics problem of this robot with at most two real solutions; the Cartesian positions of the moving platform are shown in Table 1 as $\mathbf{p}_{1}=\left(x, y, z^{1}\right)^{T}$ and $\mathbf{p}_{2}=\left(x, y, z^{2}\right)^{T}$. The solutions depend only on the geometric parameters and the lengths of the prismatic actuators $l_{1}, l_{2}$ and $l_{3}$; all double solutions lead to two configurations. Fig. 2 shows all distinct real configurations of this robot. 


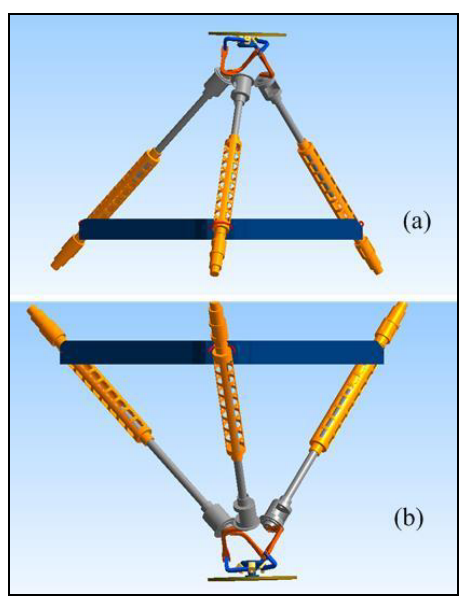

Figure 2. The two solutions of the FPKs for two configurations of the TLPM

In this project, as shown in Fig. 3, the workspace of the TLPM robot is defined as a region of the three dimensional space generated by the three prismatic joints in the FCS frame.

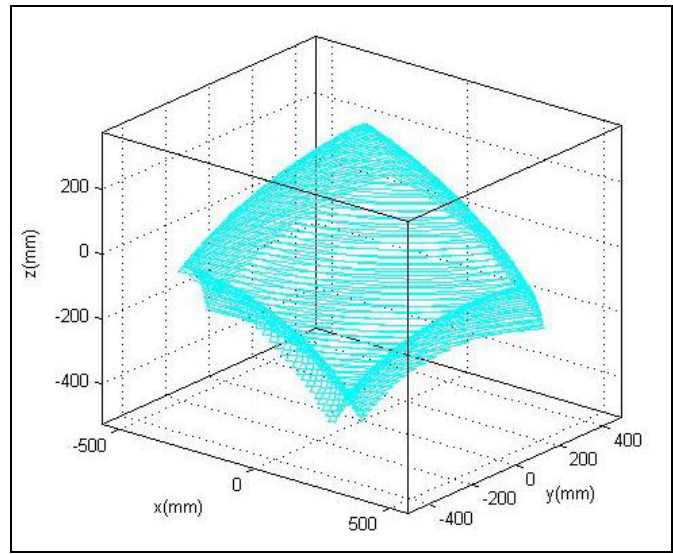

Figure 3. Feasible workspace of the TLPM

\section{Singularity}

\subsection{Jacobian matrices}

The Jacobian matrix is defined as the matrix map between of the velocity of the end effector and the vector of linear actuated joint rates. Using Jacobian matrices, singularity analysis and various kinds of singularities for the TLPM robot will be investigated.

The kinematic constraint equations, Eq. (2), are defined in the base frame FCS. Therefore, for $i^{\text {th }}$ limb of the TLPM robot, both sides of the Eq. (2) can be time differentiated to yield

$$
\mathbf{J}_{d i r} \dot{\mathbf{x}}=\mathbf{J}_{i n v} \mathbf{i}
$$

where $\dot{\mathbf{x}}=\left[\begin{array}{lll}v_{x} & v_{y} & v_{z}\end{array}\right]^{T}$ and $\mathbf{i}=\left[\begin{array}{lll}l_{1} & l_{2} & l_{3}\end{array}\right]^{T}$ are the vectors of the linear actuated joint rates and the end effector velocities, respectively. Therefore, we can get

$$
\mathbf{J}_{d i r}=\left[\begin{array}{l}
\frac{q_{1}-p}{\left\|q_{1}-p\right\|} \\
\frac{q_{2}-p}{\left\|q_{2}-p\right\|} \\
\frac{q_{3}-p}{\left\|q_{3}-p\right\|}
\end{array}\right]_{3 \times 3}, \mathbf{J}_{i n v}=\mathbf{I}_{3 \times 3}
$$

where $\mathbf{J}_{d i r}$ and $\mathbf{J}_{i n v}$ are direct and inverse Jacobian matrices, respectively. In view of Eq. (7), we can rewrite

$$
\dot{\mathbf{I}}=\mathbf{J}^{-1}{ }_{i n v} \mathbf{J}_{d i r} \dot{\mathbf{X}}
$$

Where $\mathbf{J}=\mathbf{J}^{-1}{ }_{i n} \mathbf{J}_{\text {dir }}$ is a $3 \times 3$ matrix called over all Jacobian matrices of the TLPM robot.

\subsection{Singularity analysis}

In singular configuration, the mobile platform may instantaneously gain one or more unconstrained degrees of freedom and the performances of the robot degenerate and the structure may be damaged [15]. And singularity limits the workspace of a robot. Therefore, a usable robot workspace may be obtained by avoiding such singulars from the theoretical workspace. The employed method consists the analysis of the two Jacobian matrices to determine these singular configurations.

\subsubsection{Inverse kinematic Singularity}

It is known that a parallel manipulator is in an inverse singularity when the inverse Jacobian matrix equals to zero. As shown in Eq. (8), since the matrix $\mathbf{J}_{i n v}$ is a unit matrix

$$
\mathbf{J}_{i n v}=\mathbf{I}_{3 \times 3}=\left[\begin{array}{ccc}
1 & 0 & 0 \\
0 & 1 & 0 \\
0 & 0 & 1
\end{array}\right], \operatorname{det}\left(\mathbf{J}_{i n v}\right) \neq 0
$$

Thus the inverse singularity does not occur for the TLPM robot.

\subsubsection{Direct kinematic singularity}

Direct singularity for the TLPM occurs when $\operatorname{det}\left(\mathbf{J}_{d i r}\right)=0$, based on Eq. (8), we can re-written as

$$
\begin{gathered}
\mathbf{J}_{d i r}=\left[\begin{array}{ccc}
\frac{x_{1}-x}{u_{1}} & \frac{y_{1}-y}{u_{1}} & \frac{z_{1}-z}{u_{1}} \\
\frac{x_{2}-x}{u_{2}} & \frac{x_{2}-y}{u_{2}} & \frac{x_{2}-z}{u_{2}} \\
\frac{x_{3}-x}{u_{3}} & \frac{x_{3}-y}{u_{3}} & \frac{x_{3}-z}{u_{3}}
\end{array}\right] \\
\operatorname{det}\left(\boldsymbol{J}_{d i r}\right)=\frac{s_{d}}{2 \prod_{i=1}^{3} \sqrt{u_{i}}}, i=1,2,3
\end{gathered}
$$

where $s_{d}=-3 \sqrt{3} L^{2} h-3 \sqrt{3} L^{2} z, u_{i}=\left(x_{i}-x\right)^{2}+\left(y_{i}-y\right)^{2}+\left(z_{i}-z\right)^{2}$ 
Note that $s_{d}=0, \operatorname{det}\left(\boldsymbol{J}_{\text {dir }}\right)=0$.Therefore the second types of singularity occurs whenever the position of the end effector $z=-h$. By inspection of Eq. (12), we can see that $\lim _{p \rightarrow q_{i}} \operatorname{det}\left(\boldsymbol{J}_{\text {dir }}\right)=0, i=1,2,3$. Consequently, when $\boldsymbol{p}=\boldsymbol{q}_{i}$, means that the position of the end effector is coincide with the fixed points. This condition becomes critical when the manipulator approached to singularity configurations.

Examples of the direct singularity configurations are sketched in Fig. 4.

Direct kinematic singularities for the TLPM can be avoided by choosing values for joint variables $l_{i}, i=1,2$, 3 that result in a non-singular matrix $\boldsymbol{J}_{d i r}$. Based on solving the inverse kinematics, it is possible to select a set of solutions that avoids singular poses.

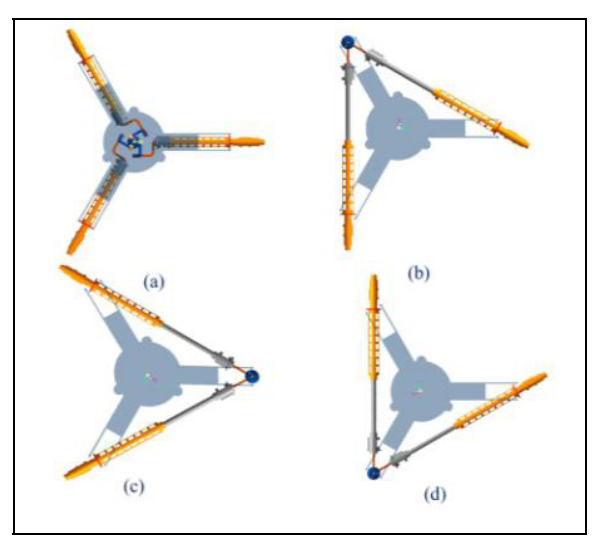

Figure 4. The two direct singularity configurations of the TLPM

\section{Link interference}

For avoiding collision, it is an essential operation before the determination of the interference among mechanical parts. The interference which might occur in TLPM can be simplified to the five situations.

The interference can be measured by the angles $\theta_{i 1}$ and $\theta_{i 2}, i=1,2,3$. For $i^{\text {th }}$ limb, the vectors from $\mathbf{p}$ to $\mathbf{q}_{i}$ denoted as $\boldsymbol{z}_{i 1}$, thus $\boldsymbol{z}_{i 1}$ can be computed as

$$
\boldsymbol{z}_{i 1}=\mathbf{q}_{i}-\mathbf{p}, i=1,2,3
$$

and its unit vectors are given by

$$
\hat{\boldsymbol{z}}_{i 1}=\frac{\mathbf{q}_{i}-\mathbf{p}}{\left\|\boldsymbol{z}_{i 1}\right\|}
$$

The vectors $\mathrm{y}_{i 1}$ can be computed as

$$
\mathbf{y}_{i 1}=\mathbf{R}\left(\boldsymbol{z}, \eta_{i 1}\right) \mathbf{y}, i=1,2,3
$$

where $\mathbf{y}=\left(\begin{array}{lll}0 & 1 & 0\end{array}\right)^{\mathrm{T}}$.

Finally, the vectors $\boldsymbol{x}_{i 1}$ are perpendicular to both of $y_{i 1}$-axis and $z_{i 1}$-axis, which can be computed as

$$
\mathbf{x}_{i 1}=\mathbf{y}_{i 1} \times \mathbf{z}_{i 1}, i=1,2,3
$$

and its unit vectors are given as

$$
\hat{\mathbf{x}}_{i 1}=\frac{\mathbf{y}_{i 1} \times \mathbf{z}_{i 1}}{\left\|\mathbf{x}_{i 1}\right\|}
$$

Therefore, the rotation matrices associated with each limb from the frame $\mathrm{L}_{i 1} \mathrm{CF}$ to the frame FCS, which are denoted as $\mathbf{R}_{i 0}$, and given as

$$
\mathbf{R}_{i 0}=\left[\begin{array}{lll}
\hat{\mathbf{x}}_{i 1} & \hat{\mathbf{y}}_{i 1} & \hat{\mathbf{z}}_{i 1}
\end{array}\right], i=1,2,3
$$

Referring to the Figure 3, the rotation matrices from frame $\mathrm{L}_{i 1} \mathrm{CF}$ to frame $\mathrm{L}_{i 2} \mathrm{CF}$ are written as

$$
\mathbf{w}_{i}=\mathbf{R}_{i 0} \mathbf{R}_{i 1} \mathbf{R}_{i 2}, i=1,2,3
$$

where

$$
\mathbf{R}_{i 2}=\mathbf{R}_{z}\left(\theta_{1}\right) \mathbf{R}_{y}\left(\theta_{2}\right)==\left[\begin{array}{ccc}
\mathrm{c} \theta_{1} \mathrm{c} \theta_{2} & -\mathrm{s} \theta_{1} & \mathrm{c} \theta_{1} \mathrm{~s} \theta_{2} \\
\mathrm{c} \theta_{2} \mathrm{~s} \theta_{1} & \mathrm{c} \theta_{1} & -\mathrm{s} \theta_{1} \mathrm{~s} \theta_{2} \\
-\mathrm{s} \theta_{2} & 0 & \mathrm{c} \theta_{2}
\end{array}\right]
$$

Now it is easy to write the rotation matrices from the frame MCS to the frame $\mathrm{L}_{i 2} \mathrm{CF}$ as

$$
\mathbf{v}_{i}=\mathbf{R}_{z}\left(\eta_{i 1}\right) \mathbf{R}_{y}\left(\frac{\pi}{4}\right) \mathbf{R}_{x}\left(\frac{\pi}{2}\right), i=1,2,3
$$

Combine Eq. (25) and Eq. (26), we can obtain $\mathbf{w}_{i}=\mathbf{v}_{i}$, and $\mathbf{R}_{i 2}$ can be computed as

$$
\begin{aligned}
& \mathbf{R}_{i 1}=\mathbf{R}_{i 1}^{-1} \mathbf{R}_{i 0}^{-1} \mathbf{R}_{z}\left(\eta_{i 1}\right) \mathbf{R}_{y}\left(\frac{\pi}{4}\right) \boldsymbol{R}_{x}\left(\frac{\pi}{2}\right) \\
& \theta_{i 1}=-A \tan 2\left(\mathbf{R}_{i 1}(1,2), \mathbf{R}_{i 1}(2,2)\right), i=1,2,3 \\
& \theta_{i 2}=-A \tan 2\left(\mathbf{R}_{i 1}(3,1), \mathbf{R}_{i 1}(3,3)\right), i=1,2,3
\end{aligned}
$$

Corresponding to the mechanical structure design of the links, link geometry of a specified is defined. In Fig. 5, interference between the links $L_{i 3}$ with the $L_{i 2}$ in one limb is checked by studying the relative rotation of link $L_{i 3}$ with respect to the link $L_{i 2}, i=1,2,3$. In the view of the specified link geometry, the minimum angle $\theta_{i 1}$ rotation around $z_{i 2}$ axis is $\pi / 10$, and the maximum angle is $\pi$. In this situation, the interference can be avoided by limiting the $\theta_{i 1}$ as: $\pi / 10<\theta_{i 1}<\pi$. The second situation occurs when the link $L_{i 3}$ interferes with moving platform, the minimum angle $\theta_{i 2}$ rotation around $z_{i 3}$ axis to moving platform is $\pi / 6$, and the maximum angle is $\pi$. Thus, $\pi / 6<\theta_{i 2}<\pi$ represents the joint is free move without mechanical interference inside the workspace.

See Fig. 6, the third situation occurs when the link $L_{i 2}$ interferes with $L_{i 2}$ in adjacent limbs; as shown in Fig. 7, the cross-shape of the $l_{i 2}$ is a squared area, the length of a side is $a$, we can ensure that there is no interference if the minimum distance between two links following condition: $D_{z i}>\sqrt{a}, i=1,2,3$. Similarly, in the fourth situation, the $L_{i 3}$ interferes with $L_{3}$ in adjacent limbs; the minimum distance between two links following condition: $D_{s i}>\sqrt{a}$ $i=1,2$, 3. And in fifth situation occurs when the $L_{i 2}$ interferes with $L_{i 3}$ in different limbs; the minimum distance between two links following condition: $D_{z s i}>\sqrt{a}$, 
$i=1,2,3$, the interference between two links can be avoided.

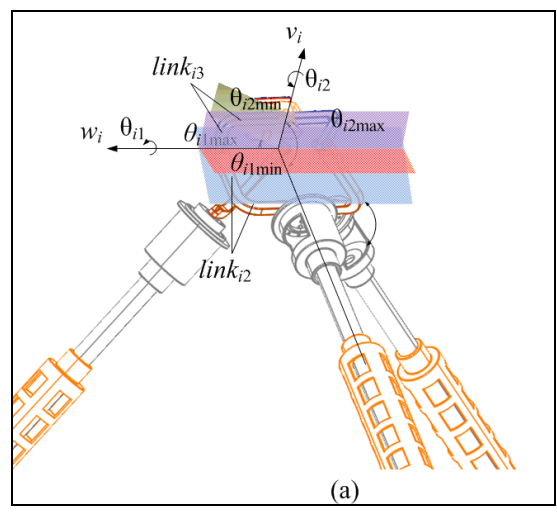

Figure 5. Angle-interference

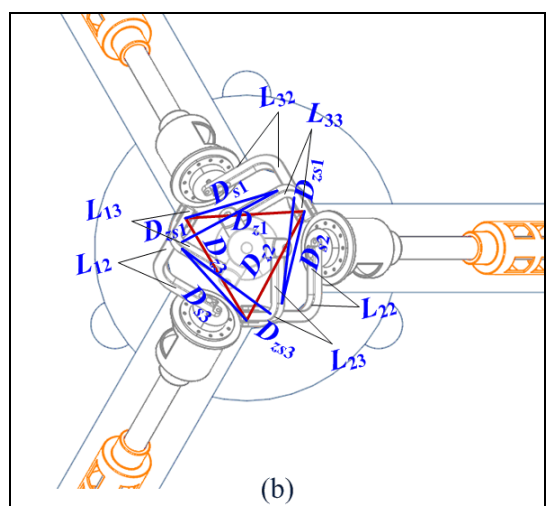

Figure 6. Distance-interference

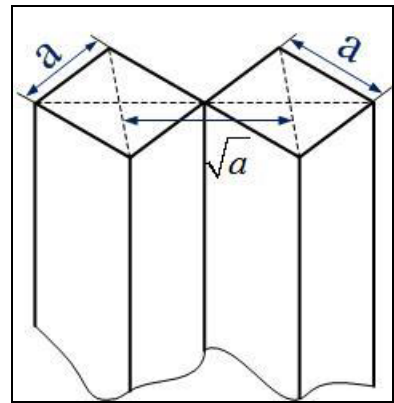

Figure 7. The distance-interference problem, the minimum safety distance between two links in the condition of noninterference

From above analysis, there are two kinds of interferences in the system, one is angle-interference in one limb, and the other is distance-interference in adjacent limbs. The angle interference for TLPM can be avoided by choosing values for joint variables $\theta_{i 1}$ and $\theta_{i 2}$ that result in non-interference angles. By modifying the shape of links can reduce interference partly.

\section{Conclusions}

This paper has presented a novel 6-DOF parallel robot. For the forward kinematic, an analytical approach yielding two position solutions are presented. The result implies that for a given set of input values, the robot can be assembled in at most two different configurations. And the reachable workspaces are determined. Next, Jacobian matrices are defined to derive the relationship between translational velocities of the moving platform and the actuated joint rates. Additionally, using pure translational Jacobian matrices, Jacobian of constraint is defined. The two types of conventional singularities are analyzed. Additionally, the angle-interference and distance-interference are presented.

And the analysis shows that the robot is free to move without mechanical singularity and interference inside the prescribed workspace. The work in this paper provides a method to easily analyze a 6-DOF parallel robot and will be a base for further optimization design of this mechanisms with clear criteria. Furthermore, the work also provides simpler kinematics model for the existing parallel mecanisms.

\section{References}

1. J. P. Merlet, ASME J. Mech. Des., 128(1), 199-206 (2006)

2. Z. Tao, \& Q. An, Mech. Mach. Theory, 69, 62-72 (2013)

3. C M Gosselin, J Sefrioui, M J Richard, ASME J. Mech. Des., 116.2: 594-598 (1994)

4. D. Gan, J. S. Dai, J. Dias, \& L. Seneviratne, ASME J. Mech. Rob,7(4), 041007, (2015)

5. J. Fu, F. Gao, , Y. Pan, , \& H. Du, Adv Mech Eng., 7(5), 1687814015582118. (2015)

6. F. Gao, B. Peng, W. Li, H. Zhao, Robotica, 23, 35$43(2005)$

7. A. Rezaei, \& A. Akbarzadeh, Mech. Mach. Theory, 86, 211-234. (2015)

8. X. J. Liu, J. Wang, J. Kim, ASME J. Mech. Des., 128(2) 365-373, (2006)

9. G. Pond, J. A, Mech. Mach. Theory, 42, 1388-1400, (2007)

10. I. A. Bonev, J. Ryu, Mech. Mach. Theory, 36, 15-28, (2001)

11. D. Chablat, P.Wenger, F. Majou, J. P. Merlet, Int. J. Rob. Res., 23, 615-624, (2004)

12. A. Rezaei, A. Akbarzadeh, P. M. Nia, M. R. Akbarzadeh-T, Robot Cim-Int Manuf., 29.4: 158-173 (2013)

13. F. irmani, F, \& R. P. odhorodeski, Mech. Mach. Theory, 44(7), 1386-1399 (2009)

14. S. C. Burgess, \& A. C. Etoundi, ASME J. Mech. Des., 136(11), 115002 (2014)

15. I. Ebrahimi, J. A. Carretero, R. Boudreau, Mech. Mach. Theory, 42(8), 1007-1016 (2007) 